\title{
Elaboración y validación de una escala de actitudes hacia la pornografía
}

\author{
MARTA MonferRer Balaguer \\ al225781@uji.es \\ PATRICIA FLOR ARASIL \\ al228961@uji.es
}

\section{Resumen}

La pornografía se ha convertido en uno de los intereses más compartido por la sociedad moderna, por lo tanto, el objetivo de este artículo es la realización de una escala que mida actitudes hacia la pornografía. El cuestionario incluyó ítems con respecto a las dimensiones de diversión, excitación, aprendizaje y norma social, referidas a la pornografía. El grupo de estudio estuvo formado por 126 sujetos (59 hombres y 67 mujeres), con edades comprendidas entre 18 y 63 años (mediana: 24 años). La consistencia interna es alta, aunque el análisis factorial confirmatorio no ha verificado nuestras dimensiones propuestas. La falta de material científico sobre las actitudes sociales hacia la pornografía sugiere la conveniencia de realizar futuros estudios exploratorios sobre el tema.

Palabras clave: escala, pornografía, actitudes, validez, fiabilidad.

\section{Abstract}

Pornography has become one of the most widespread interests in the modern society, therefore, the objective of this article is the construction of a scale that measures the attitudes towards pornography. The questionnaire contained a series of items that belonged to the dimensions enjoyment, sexual excitement, learning, and social norm. The study group was formed by 126 participants (59 men and 67 women), with ages between 18 and 63 years old (median: 24 years). Internal consistency of the scale is high, showing homogeneity between ítems. However, the factorial analysis has refused the factors we proposed. Lack of scientific material about social attitudes toward pornography suggests desirability of carrying out further exploratory studies about this topic.

Keywords: scale, pornography, attitudes, validity, reliability. 


\section{Introducción}

El porno sigue siendo un tema tabú, muy poca gente reconoce abiertamente que consume porno, pero la realidad es que la industria pornográfica está más expandida que el fútbol profesional, baloncesto y béisbol juntos (Rich, 2001). Si introducimos la palabra «porno» en el buscador de Google, el número de resultados encontrados llega a la asombrosa cantidad de 200.000.000.

Socialmente, el consumo de pornografía ha sido considerado en muchas ocasiones como el causante de distintos problemas de pareja, en adolescentes, familias, e incluso de violencia y abuso sexual (Fagan, 2009), pero con las tecnologías de la información (T. I. C.) y su anonimidad, la eliminación de los estigmas sociales y la creciente pérdida de fe religiosa, se está contribuyendo a la expansión de la industria pornográfica, siendo ya dominante en Internet (Carnes, Delmonico, y Griffin, 2001; Carroll et al., 2008).

Todas las sociedades tienen sus propias normas, a veces bastante rígidas, que moldean las actitudes sexuales de sus miembros, y es que el sexo, uno de los placeres corporales más intensos, ha tenido una connotación ambivalente en distintas culturas y en distintas épocas (García Bellaúnde Velarde, 2005). Diversas investigaciones han demostrado que los resultados obtenidos en una determinada cultura no son generalizables para todas las sociedades (Lam y Chan, 2007; Gunter, 2002), ya que existen aspectos muy relevantes en ciertas sociedades con respecto a otras que van a influenciar en gran medida el consumo de pornografía, como por ejemplo, las creencias religiosas, que pueden disminuir el visionado de pornografía (Collins et al., 2004; Wright, Bae, y Funk, 2013), y las ideológicas también han demostrado ser un factor importante: aquellas personas conservadoras la interpretan como desensibilizadora y que contribuye a efectos no deseados (Hald y Malamuth, 2008). Por lo tanto, una definición de pornografía que recoja todas estas peculiaridades podría ser la de Hald, Malamuth, y Yuen (2010), que la definen como "material sexualmente explícito con la intención de crear arousal sexual al consumidor», teniendo en cuenta que la exposición a este material es de carácter voluntario.

En cuanto a las diferencias de sexo, generalmente se ha demostrado que sí que existen. Los estudios apoyan que los hombres tienen una actitud más favorable y un primer contacto con la pornografía a edad más temprana que las mujeres (Malamuth, 1996). Los hombres se muestran satisfechos y curiosos sexualmente, sin aparentes represiones y a favor de la experimentación, en cambio, las mujeres pueden experimentar vergüenza al hablar de sexo (Ampuero, 1999) y tienen reacciones más negativas con respecto al material sexualmente explícito.

Existen gran cantidad de estudios e investigaciones que relacionan, como hemos dicho, el consumo de pornografía con actitudes religiosas, las nuevas tecnologías, las edades, el sexo Pero, ¿existe una escala fiable y válida para medir las actitudes hacia la pornografía? Lo cierto es que no hay grandes fuentes de bibliografía ni estudios al respecto como para concluir con una escala predominante que recoja todas las dimensiones y aspectos, y desde este estudio, consideramos que es necesario contar con una para poder desarrollar posteriores relaciones de este tipo de actitudes con cualquier otro aspecto. Por lo tanto, el objetivo de este trabajo es la construcción de una escala que mida las actitudes hacia la pornografía, con la finalidad de conseguir una medición correcta, para poder contar con una buena base para futuros estudios que la requieran. 


\section{Método}

\section{Participantes}

Para realizar el estudio se llevó a cabo un muestreo opinático, cuya muestra se compuso por 126 sujetos, de los cuales 59 fueron hombres y 67 mujeres, con edades comprendidas entre 18 y 63 años (mediana: 24). Los sujetos eran todos de nacionalidad española, y mayoritariamente de la Comunidad Valenciana.

\section{Instrumentos}

La difusión de la escala elaborada con los 49 ítems se llevó a cabo mediante un formulario de Google Docs, el cuál se dio a conocer a través de redes sociales y foros de todo tipo.

Los posteriores análisis estadísticos y resultados se llevaron a cabo mediante el programa estadístico IBM SPSS Statistics 21.0 y AMOS.

\section{Procedimiento}

Tras la documentación previa sobre pornografía, extraímos cuatro dimensiones que consideramos agrupaban la totalidad de las actitudes: diversión, excitación, curiosidad y aceptación social. Posteriormente, se redactaron una serie de ítems que englobaran cada factor, con la ayuda de las respuestas que nos ofrecieron a ciertas preguntas realizadas en distintos foros de todo tipo de temáticas (no sólo de sexualidad), para evitar cualquier tipo de sesgo. Una vez tuvimos un número suficiente de ítems para cada dimensión (5 ó 6 aproximadamente), invertimos algunos de ellos, y los juntamos formando la escala inicial.

Esta escala de actitudes hacia la pornografía, inicialmente constaba de 49 ítems, que se administraron a través de un formato de Google Docs, introduciendo unas breves instrucciones al inicio. Este programa permitía inhabilitar las respuestas en blanco, no permitiendo enviar la escala si no estaban todos los ítems respondidos.

La escala fue dada a conocer y ofrecida voluntariamente a los participantes mediante publicaciones en redes sociales, foros, y vía mail. Es por ello que la colaboración en la investigación era voluntaria, anónima y no ofrecía ninguna recompensa para los evaluados.

Una vez obtuvimos los datos de una muestra representativa, se llevó a cabo un análisis de fiabilidad de las contestaciones mediante el programa estadístico IBM SPSS Statistics 21.0. La matriz de correlaciones total-elemento nos ayudó a establecer un criterio con el que finalmente redujimos la escala, para volver a llevar a cabo un segundo análisis de fiabilidad Alfa de Cronbach, y un análisis factorial confirmatorio del modelo propuesto mediante el AMOs.

\section{Resultados}

Una vez se obtuvieron las contestaciones de la escala para una muestra suficiente (126 personas), se comprovó la fiabilidad y validez de la misma. Para comprobar la fiabilidad total de la escala, se realizó el cálculo del Alfa de Cronbach, que resultó ser de 0,95 en su versión original de 49 ítems. De este modo, una vez eliminados aquellos ítems (equivalentes a otros) con menor fiabilidad, la escala quedó reducida a 32 ítems, y el Alfa de Cronbach se redujo a 0,943 (ver Tabla 1). 
Posteriormente, se analizó también la fiabilidad para cada uno de los factores establecidos en nuestra escala, siendo éstos los siguientes:

- Factor 1. Las preguntas agrupadas en este Factor hacen referencia a Diversión: ítems $3,5,6,15,19,20,21,25,31$.

Diversión: hace referencia a al entretenimiento, aburrimiento o distracción.

- Factor 2. Las preguntas agrupadas en este Factor hacen referencia a Excitación: ítems $7,8,22,23,29,34,35$.

Excitación: corresponde a manifestaciones orgánicas que generan un aumento en el libido sexual.

- Factor 3. Las preguntas agrupadas en este Factor hacen referencia a Curiosidad: ítems $18,27,28,30,48,49$.

Curiosidad: dimension referida al aparendizaje, exploración y mejora de prácticas sexuales.

- Factor 4. Las preguntas agrupadas en este Factor hacen referencia a Aceptación Social: ítems 16,17,32,36,37,38,40,42,46,47.

Aceptación Social: tiene como significado la influencia de la norma social, la influencia de la deseabilidad social.

\begin{tabular}{llcc}
\hline & & ALFA DE & N DE \\
CRONBACH & ELEMENTOS \\
\hline \multirow{2}{*}{ Inicial } & &, 943 & 32 \\
& &, 950 & 49 \\
& Diversión / Aburrimiento &, 855 & 9 \\
& Excitación / Deseo &, 827 & 7 \\
& Curiosidad Aprendizaje &, 759 & 6 \\
& Influencia / Aceptación Social &, 885 & 10 \\
\hline
\end{tabular}

Para comprobar la validez de la escala reducida, se realizó un análisis factorial confirmatorio, para ver si el modelo factorial propuesto era correcto (ver Figura 1), y permitía establecer relaciones causales mediante ecuaciones estructurales, combinando la eficacia de cada subescala con el error. A pesar de lo esperado, los resultados obtenidos mediante el programa AMOS, no confirmaron que el modelo fuera válido para medir las actitudes hacia la pornografía, tal y como puede observarse en la Tabla 3. Los índices de ajuste para los modelos en NFI, RFI, IFI, TLI, y CFI, resultaron ser menores al valor óptimo 0,90 (Hu y Bentler, 1999), al cual deberían haber igualado o superado para considerar que ajustaban de forma correcta. Por lo que respecta al índice de error RMSEA, éste también mostró un valor elevado e inadecuado con respecto al 0,05 máximo de referencia para aceptar el modelo (Hu y Bentler, 1999). 
Tabla 2

Comparaciones de línea base

\begin{tabular}{|c|c|c|c|}
\hline & DEFAULT MODEL & SATURATED MODEL & INDEPENDENCE MODEL \\
\hline NFI & ,647 & 1,000 &, 000 \\
\hline RFI & ,619 & & 000 \\
\hline IFI & ,776 & 1,000 & ,000 \\
\hline TLI & ,754 & &, 000 \\
\hline CFI & ,772 & 1,000 & ,000 \\
\hline RMSEA & ,095 & & , 191 \\
\hline LO 90 & ,086 & & , 184 \\
\hline HI 90 & 103, & & 198, \\
\hline
\end{tabular}

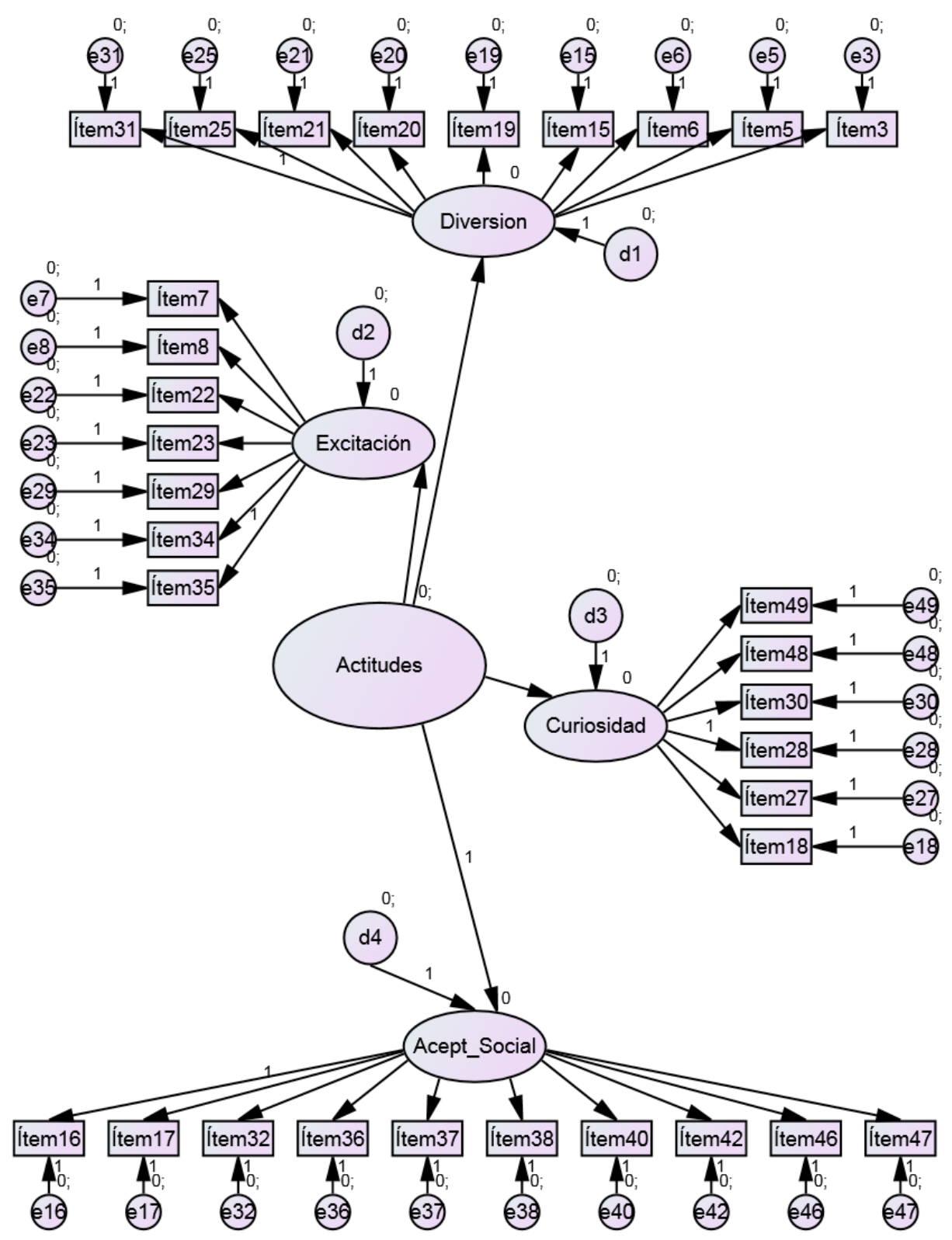

Figura 1. Modelo factorial de la Escala de Actitudes hacia la Pornografía 


\section{Discusión}

El objetivo del presente estudio era la construcción de un instrumento de medida, concretamente una escala, que midiese las actitudes hacia la pornografía. La escala inicial era muy fiable, tal y como se había previsto, ya que se había formado con ítems exclusivamente elaborados y sus inversos, formando una totalidad de 49 ítems. Pero la finalidad de empezar con una escala tan completa, era comprobar cuáles correlacionaban mejor con la escala total, para quedarnos con los equivalentes mejores. De este modo, la escala final estuvo compuesta por un total de 32 ítems, cuyo formato de respuesta fue una escala Likert de 5 alternativas que iban desde «completamente en desacuerdo» hasta "completamente de acuerdo» (Ver Anexo). El criterio utilizado para dicha reducción, fue la eliminación de aquellos ítems que obtuvieron menores valores en el análisis de fiabilidad.

Los resultados estadísticos que hemos obtenido para la escala, una vez fue reducida, reflejan unos índices de fiabilidad altos, es decir, los ítems representan de forma adecuada y precisa la actitud general hacia la pornografía. Por otro lado, los resultados del análisis de fiabilidad obtenidos mediante el Alfa de Cronbach para las 4 dimensiones propuestas (diversión, excitación, aprendizaje y aceptación social) fueron un poco más bajos que en la escala total (ver Tabla 1), ya que al especificar en cada dimensión, es más complicado obtener alta fiabilidad, cosa que no ocurre cuando se mide la fiabilidad de una escala global de actitudes, que abarca mayor rango de aspectos. Aún así, se trata de índices altos, que confirman que las facetas son fiables en la medición.

Por lo que respecta a la validez ha resultado ser baja. Los resultados obtenidos mediante el programa AMOS, no confirmaron que el modelo fuera válido para medir las actitudes hacia la pornografía, pues éste no se ajusta a la medición de las actitudes referidas a la pornografía tal y como se esperaba. Esta falta de validez, se atribuye a la limitación de la muestra, considerando que se trata de una escala con la que se puede seguir trabajando.

En cuanto a limitaciones, cabe destacar que aunque la fiabilidad nos ha resultado muy alta, no existen unas condiciones de evaluación óptimas que garanticen una fiabilidad perfecta, y por más que el cuestionario sea anónimo, siempre habrá cierto error de medida por resistencia a lidiar con los temas sexuales, sobre todo por lo que se refiere a la deseabilidad social y la influencia de lo que se considera socialmente correcto.

La pornografía es un tema actual con una creciente expansión en la sociedad. A pesar de su popularidad temática, se sigue considerando un tema tabú y no existe gran cantidad de estudios que permitan hacer un análisis desde el punto de vista científico, ni una escala definitiva para su medición. Por esto, consideramos importante la construcción de un instrumento fiable y válido que permita describir el posicionamiento de las personas respecto a sus actitudes hacia la pronografía. Además de permitir mayor investigación, es importante destacar su aplicación práctica y su utilidad en el contexto clínico, como herramienta auxiliar para la descripción y diagnóstico de posibles trastornos sexuales.

Como recomendaciones para posibles replicaciones, se recomienda ampliar la muestra de tipificación para que ésta sea más representativa, y conseguir así, que al comprobar la validez con un análisis factorial confirmatorio, el modelo aprueve las dimensiones planificadas inicialmente, tal y como se esperaba, ya que la limitación de la muestra ha sido considerada como la causa del desajuste. 


\section{Agradecimiento}

Agradecer enormemente a Francisco Herrero Machancoses, profesor asociado de la Universidad Jaime I de Castellón, por su ayuda prestada en la elaboración de la escala y la edición del artículo.

\section{Referencias bibliográficas}

Ampuero, A. (1999). Sexualidad y deseo: hablan adolescentes de Ayacucho, Puno, San Martín y Ucayali. Perú: Movimiento Manuela Ramos.

Carnes, P., Delmonico, D. L., y Griffin, E. (2001). In the shadows of the Net. Center City, MN: Hazelden.

Carroll, J. S., Padilla-Walker, L. M., Nelson, L. J., Olson, C. D., McNamara Barry, C., y Madsen, S. D. (2008). Generation XXX: Pornography Acceptance and Use Among Emerging Adults. Journal of Adolescent Research, 23(1), 6-30. doi:10.1177/0743558407306348

Collins, R. L., Elliott, M. N., Berry, S. H., Kanouse, D. E., Kunkel, D., Hunter, S. B., y Miu, A. (2004). Watching sex on television predicts adolescent initiation of sexual behavior. Pediatrics, 114(3), 280-289. doi:10.1542/peds.2003-1065-L

Fagan, P. F. (2009). The Effects of Pornography on Individuals, Marriage , Family , and Community.

García Bellaúnde Velarde, V. A. (2005). Actitudes sexuales en adolescentes universitarios. Universidad de Lima.

Gunter, B. (2002). Media sex. Mahwah, NJ: Lawrence Erlbaum.

Hald, G. M., y Malamuth, N. M. (2008). Self-perceived effects of pornography consumption. Archives of sexual behavior, 37(4), 614-25. doi:10.1007/s10508-007-9212-1

Hald, G. M., Malamuth, N. M., y Yuen, C. (2010). Pornography and attitudes supporting violence against women: revisiting the relationship in nonexperimental studies. Aggressive Behavior, 36(1), 14-20. doi:10.1002/ab.20328

Lam, C. B., y Chan, D. K.-S. (2007). The use of cyberpornography by young men in Hong Kong: some psychosocial correlates. Archives of sexual behavior, 36(4), 588-98. doi:10.1007/ s10508-006-9124-5

Hu, L., Bentler, P. M. (1999) Cutoff criteria for fit indexes in covariance structure analysis: Conventional criteria versus new alternatives. Structural Equation Modeling: A Multidisciplinary Journal, 6(1), 1-55.

Malamuth, N. M. (1996). Sexually Explicit Media, Gener Differences, and Evolutionary Theory. Journal of communication, 46(3), 8-31.

Rich, F. (2001). Naked capitalists. In M. Stombler, E. O. Burgess, D. Donnelly, y W. Simonds (eds.), Sex matters: The sexuality and society reader (pp. 462-466). Boston: Pearson Education Inc.

Wright, P. J., Bae, S., y Funk, M. (2013). United States Women and Pornography Through Four Decades: Exposure, Attitudes, Behaviors, Individual Differences. Archives of sexual behavior, 42, 1131-1144. doi:10.1007/s10508-013-0116-y 


\section{Anexo}

\section{Escala de actitudes hacia la pornografía (versión preliminar)}

\section{Instrucciones}

A continuación le presentaremos una serie de preguntas que nos servirán para clasificar la información que nos dé, y poder establecer conclusiones más pormenorizadas.

\begin{tabular}{ll}
\hline Sexo: M/F & Estado Civil: \\
Edad: & a) Soltero/a \\
Población: & b) Con pareja \\
Provincia: & c) Casado/a o viviendo en pareja \\
& d) Divorciado/a
\end{tabular}

Nacionalidad:

Nivel de estudios:

a) Estudios primarios

Profesión:

b) Graduado Escolar
a) Estudiante
b) Parado
c) Trabajador por cuenta ajena
d) Autónomo
e) Jubilado

c) Bachillerato

d) Formación Profesional

e) Estudios universitarios/ superiores

f) Otros (especificar):

Por favor, antes de comenzar, lea cuidadosamente estas instrucciones. Esta escala consta de 49 frases. Lea cada frase con atención y marque la alternativa que refleje mejor su acuerdo o desacuerdo con lo que dice la frase. Señale:

- Completamente en desacuerdo, si la frase es completamente falsa en su caso.

- Desacuerdo, si la frase es frecuentemente falsa.

- Indiferente, si la frase es tan cierta como falsa, si no puede decidirse, o si Vd. se considera neutral en relación con lo que se dice en ella.

- De acuerdo, si la frase es frecuentemente cierta.

- Completamente de acuerdo, si la frase es completamente cierta.

No hay respuestas correctas ni incorrectas, y no se necesita ser un experto para contestar a esta Escala. Conteste de forma sincera y exprese sus opiniones de la manera más precisa posible.

Dé una respuesta a todas las frases. Si se equivoca o cambia de opinión, cambie la opción escogida anteriormente. Recuerde que los resultados obtenidos son totalmente anónimos e intransferibles.

1. La pornografía es un medio para evadir los problemas.

2. La pornografía es inútil.

3. La pornografía me divierte.

4. La pornografía me divierte.

5. La pornografía no me resulta placentera.

6. La pornografía es una buena distracción en mis horas libres.

7. La pornografía me excita.

8. Incluyo la pornografía en mi relación de pareja.

9. Ver pornografía antes de una relación sexual no tiene ningún sentido. 
10. Ver pornografía no es un buen método para estimularse.

11. La pornografía no influye en mis sentimientos hacia una relación sexual.

12. La pornografía no me pone.

13. La pornografía ayuda a aumentar mis conocimientos sobre el sexo.

14. Me gusta conocer más formas de practicar sexo.

15. La pornografía me aburre.

16. La gente debería ver más pornografía.

17. La pornografía es una vergüenza social

18. La pornografía crea expectativas irreales.

19. La pornografía me da placer.

20. La pornografía no es un buen medio para dejar a un lado los problemas.

21. Ver pornografía me hace sentir culpable.

22. La pornografía es un buen método para estimularse antes de una relación sexual.

23. La pornografía elimina sentimientos de las propias relaciones sexuales.

24. La pornografía simula una relación sexual de hoy en día.

25. Ver pornografía es una pérdida de tiempo.

26. Se aprenden prácticas sexuales viendo pornografía.

27. La pornografía aumenta mi interés en saber más sobre sexo.

28. El visionado de pornografía ayuda a mejorar las prácticas sexuales.

29. La pornografía es asquerosa.

30. La pornografía no me enseña nada nuevo.

31. La pornografía me alegra.

32. La pornografía es una degradación del acto sexual.

33. En la pornografía tanto los hombres como las mujeres son tratados por igual.

34. La pornografía me atrae.

35. Ver pornografía es un buen método para estimularse en solitario.

36. La pornografía debería ser ilegal.

37. Ver pornografía es como serle infiel a la pareja.

38. Quien ve pornografía es un/a pervertido/a.

39. La pornografía no tiene ningún peligro.

40. Ver pornografía es algo normal.

41. La pornografía enciende el deseo sexual.

42. La pornografía es peligrosa.

43. La pornografía no es un tipo de infidelidad.

44. La pornografía no hace daño a nadie.

45. La pornografía no favorece el aprendizaje de prácticas sexuales.

46. La pornografía es una falta de respeto.

47. La pornografía discrimina a la mujer.

48. La pornografía me hace sentir más seguro de mi mismo.

49. La pornografía ayuda a perder prejuicios hacia el sexo. 


\section{Escala de actitudes hacia la pornografía (versión definitiva, con los ítems redistribuidos)}

\section{Instrucciones}

A continuación le presentaremos una serie de preguntas que nos servirán para clasificar la información que nos dé, y poder establecer conclusiones más pormenorizadas.

\begin{tabular}{|lc|}
\hline Sexo: M/F & Estado Civil: \\
Edad: & f) Soltero/a \\
Población: & g) Con pareja \\
Provincia: & h) Casado/a o viviendo en pareja \\
Nacionalidad: & i) Divorciado/a \\
Nivel de estudios: & j) Viudo/a \\
g) Estudios primarios & \\
h) Graduado Escolar & Profesión: \\
i) Bachillerato & f) Estudiante \\
j) Formación Profesional & g) Parado \\
k) Estudios universitarios/ superiores & h) Trabajador por cuenta ajena \\
I) Otros (especificar): & i) Autónomo \\
\hline
\end{tabular}

Por favor, antes de comenzar, lea cuidadosamente estas instrucciones. Esta escala consta de 49 frases. Lea cada frase con atención y marque la alternativa que refleje mejor su acuerdo o desacuerdo con lo que dice la frase. Señale:

- Completamente en desacuerdo, si la frase es completamente falsa en su caso.

- Desacuerdo, si la frase es frecuentemente falsa.

- Indiferente, si la frase es tan cierta como falsa, si no puede decidirse, o si Vd. se considera neutral en relación con lo que se dice en ella.

- De acuerdo, si la frase es frecuentemente cierta.

- Completamente de acuerdo, si la frase es completamente cierta.

No hay respuestas correctas ni incorrectas, y no se necesita ser un experto para contestar a esta Escala. Conteste de forma sincera y exprese sus opiniones de la manera más precisa posible.

Dé una respuesta a todas las frases. Si se equivoca o cambia de opinión, cambie la opción escogida anteriormente. Recuerde que los resultados obtenidos son totalmente anónimos e intransferibles.

1. La pornografía me divierte.

2. La pornografía no me resulta placentera.

3. La pornografía es una buena distracción en mis horas libres.

4. La pornografía me excita.

5. Incluyo la pornografía en mi relación de pareja.

6. La pornografía me aburre.

7. La gente debería ver más pornografía.

8. La pornografía es una vergüenza social

9. La pornografía crea expectativas irreales. 
10. La pornografía me da placer.

11. La pornografía no es un buen medio para dejar a un lado los problemas.

12. Ver pornografía me hace sentir culpable.

13. La pornografía es un buen método para estimularse antes de una relación sexual.

14. La pornografía elimina sentimientos de las propias relaciones sexuales.

15. Ver pornografía es una pérdida de tiempo.

16. La pornografía aumenta mi interés en saber más sobre sexo.

17. El visionado de pornografía ayuda a mejorar las prácticas sexuales.

18. La pornografía es asquerosa.

19. La pornografía no me enseña nada nuevo.

20. La pornografía me alegra.

21. La pornografía es una degradación del acto sexual.

22. La pornografía me atrae.

23. Ver pornografía es un buen método para estimularse en solitario.

24. La pornografía debería ser ilegal.

25. Ver pornografía es como serle infiel a la pareja.

26. Quien ve pornografía es un/a pervertido/a.

27. Ver pornografía es algo normal.

28. La pornografía es peligrosa.

29. La pornografía es una falta de respeto.

30. La pornografía discrimina a la mujer.

31. La pornografía me hace sentir más seguro de mi mismo.

32. La pornografía ayuda a perder prejuicios hacia el sexo. 